\title{
ADAPTATION OF REPAIRED RESIN COMPOSITE RESTORATIONS USING SONICFILL COMPOSITE
}

\author{
Nelly Hussein Sabry Saada*, Mai Mahmoud Yousry** and Mohamed Fouad Haridy ${ }^{* * *}$
}

\begin{abstract}
This study was done to determine the number of voids present in repaired resin composite restoration using different types of composite. M \& M: 60 premolars were selected, Class I cavity preparation was done having two standardized mesial and distal defect to be further repaired. Teeth were aged for 1 month then followed by the repair procedure. Teeth were divided into four group according to surface treatment $\left(\mathrm{S}_{0}\right)$ no surface treatment, $\left(\mathrm{S}_{1}\right)$ Etching only, $\left(\mathrm{S}_{2}\right)$ was etch-and-rinse while $\left(\mathrm{S}_{3}\right)$ self-etch group. Then repair was done using conventional composite (M1), flowable composite (M2), and sonicfill composite (M3). Teeth were cut into two halves using Isomet saw to be further examined under Digital Handheld Light Microscope. Conclusion: A statistical significant difference was found between different materials with no surface treatment with $p$ value $<0.005$, while no statistical significant difference with other surface treatments with different materials.
\end{abstract}

\section{INTRODUCTION}

Direct resin composite restorations have been largely employed to restore posterior teeth due to their low cost and less need for the removal of sound tooth substance when compared to indirect restorations, as well as to their ease of application and acceptable clinical performance. ${ }^{(1)}$ Other alternative treatments, such as repair or resurfacing, increase the longevity of restorations at a lower cost than that of replacement, and they are the most conservative option. ${ }^{(2)}$ Marginal defects often necessitate the use of a flowable repair material to achieve proper adaptation. However, the strength properties of such materials might not be suitable for interfacial stresses induced at cavity preparation margin at stress bearing areas. It also depend on the surface treatment either chemically or mechanically that was advocated into the repair site.

\section{MATERIALS AND METHODS}

A total number of sixty freshly extracted, intact, non-carious and human premolars were assigned for this study. Standardized, box-shaped Class I occlusal cavities of $3 \mathrm{~mm}$ depth, $3 \mathrm{~mm}$

* Demonstrator, Operative \& Esthetics Department, Faculty of Oral \& Dental Medicine British University, Cairo, Egypt,

** Professor of Operative Dentistry, Faculty of Oral and Dental Medicine, Cairo University

*** Associate Professor of Operative Dentistry, Faculty of Oral and Dental Medicine, Cairo University 
bucco-lingual width and $4 \mathrm{~mm}$ mesio-distal width were prepared. The prepared cavities were restored using a universal resin composite material; Z350 XT (3M ESPE, St Paul, MN, USA) B3 Shade. The restorative procedure was done following the manufacturer's instructions. Two objects were placed inside the cavity at the mesial and distal walls after bonding protocol was done. Teeth were subjected to a period of one month for pre-repair aging of teeth in a saline solution at $37 \mathrm{C}$ in an incubator (TITANOX, Italy). Teeth were divided into four equal groups (15 each), according to the pretreatment protocol used before application of the repair resin composite $(\mathrm{S})$, where the first subgroup $\left(\mathrm{S}_{0}\right)$ was left without surface treatment (Control), the second subgroup $\left(\mathrm{S}_{1}\right)$ was subjected to etching only, $\left(\mathrm{S}_{2}\right)$ application of etch-and-rinse adhesive system and $\left(\mathrm{S}_{3}\right)$ application of self-etch adhesive system. Then, each subgroup was divided into three classes (5 each) according to the repair material used $(\mathrm{M})$; either SonicFill composite $\left(\mathrm{M}_{1}\right)$, flowable composite $\left(\mathrm{M}_{2}\right)$ or conventional composite $\left(\mathrm{M}_{3}\right)$. Teeth were cut into two halves using Isomet saw to be further examined under Digital Handheld Light Microscope under 70X magnification to detect any voids present within the repaired restoration. Image interpretation was done using image $\mathrm{J}$ software.

\section{RESULTS}

Data of voids of the repair material showed nonparametric distribution, so Kruskal Wallis test was used to compare between repair materials within each surface treatment and within each of the repair materials.

\section{Effect of different repair materials within each surface treatment and Substrate condition:}

Frequency and Percentage for the voids count for difference repair materials within each surface treatment and Substrate condition were presented in table (1) and figure (1).

TABLE (1) Frequency and Percentage for the voids count between different repair materials within each surface treatment and Substrate condition:

\begin{tabular}{|c|c|c|c|c|c|c|c|c|c|}
\hline & \multirow{3}{*}{\multicolumn{2}{|c|}{ Sonic Fill }} & \multicolumn{6}{|c|}{ Repair material } & \multirow{3}{*}{ p-value } \\
\hline & & & \multicolumn{2}{|c|}{ Flowable } & \multicolumn{2}{|c|}{ Conventional } & \multirow[b]{2}{*}{$\mathrm{N}$} & \multirow[b]{2}{*}{$\%$} & \\
\hline & & & $\mathrm{N}$ & $\%$ & $\mathrm{~N}$ & $\%$ & & & \\
\hline \multirow{15}{*}{$\begin{array}{l}\text { No of } \\
\text { Voids }\end{array}$} & \multirow{8}{*}{ Without ttt } & .00 & 4 & $100.0 \%$ & 2 & $50.0 \%$ & 0 & $0.0 \%$ & \multirow{8}{*}{$0.019^{*}$} \\
\hline & & 1.00 & 0 & $0.0 \%$ & 1 & $25.0 \%$ & 0 & $0.0 \%$ & \\
\hline & & 2.00 & 0 & $0.0 \%$ & 0 & $0.0 \%$ & 0 & $0.0 \%$ & \\
\hline & & 3.00 & 0 & $0.0 \%$ & 0 & $0.0 \%$ & 0 & $0.0 \%$ & \\
\hline & & 4.00 & 0 & $0.0 \%$ & 0 & $0.0 \%$ & 1 & $25.0 \%$ & \\
\hline & & 5.00 & 0 & $0.0 \%$ & 1 & $25.0 \%$ & 1 & $25.0 \%$ & \\
\hline & & 6.00 & 0 & $0.0 \%$ & 0 & $0.0 \%$ & 2 & $50.0 \%$ & \\
\hline & & Rank & \multicolumn{2}{|c|}{$\mathrm{a}$} & \multicolumn{2}{|c|}{$\mathrm{a}$} & \multicolumn{2}{|c|}{$\mathrm{b}$} & \\
\hline & \multirow{7}{*}{ Etching } & .00 & 2 & $50.0 \%$ & 0 & $0.0 \%$ & 1 & $25.0 \%$ & \multirow{7}{*}{$0.195 \mathrm{NS}$} \\
\hline & & 1.00 & 1 & $25.0 \%$ & 0 & $0.0 \%$ & 1 & $25.0 \%$ & \\
\hline & & 2.00 & 0 & $0.0 \%$ & 2 & $50.0 \%$ & 1 & $25.0 \%$ & \\
\hline & & 3.00 & 1 & $25.0 \%$ & 1 & $25.0 \%$ & 1 & $25.0 \%$ & \\
\hline & & 4.00 & 0 & $0.0 \%$ & 1 & $25.0 \%$ & 0 & $0.0 \%$ & \\
\hline & & 5.00 & 0 & $0.0 \%$ & 0 & $0.0 \%$ & 0 & $0.0 \%$ & \\
\hline & & 6.00 & 0 & $0.0 \%$ & 0 & $0.0 \%$ & 0 & $0.0 \%$ & \\
\hline
\end{tabular}




\begin{tabular}{|c|c|c|c|c|c|c|c|c|c|}
\hline & \multirow{3}{*}{\multicolumn{2}{|c|}{ Sonic Fill }} & \multicolumn{6}{|c|}{ Repair material } & \multirow{3}{*}{ p-value } \\
\hline & & & \multicolumn{2}{|c|}{ Flowable } & \multicolumn{2}{|c|}{ Conventional } & \multirow[b]{2}{*}{$\mathrm{N}$} & \multirow[b]{2}{*}{$\%$} & \\
\hline & & & $\mathrm{N}$ & $\%$ & $\mathrm{~N}$ & $\%$ & & & \\
\hline \multirow{14}{*}{$\begin{array}{l}\text { No of } \\
\text { Voids }\end{array}$} & \multirow{7}{*}{$\begin{array}{l}\text { Etch-and- } \\
\text { rinse }\end{array}$} & .00 & 0 & $0.0 \%$ & 2 & $50.0 \%$ & 1 & $25.0 \%$ & \multirow{7}{*}{$0.923 \mathrm{NS}$} \\
\hline & & 1.00 & 2 & $50.0 \%$ & 0 & $0.0 \%$ & 1 & $25.0 \%$ & \\
\hline & & 2.00 & 1 & $25.0 \%$ & 1 & $25.0 \%$ & 1 & $25.0 \%$ & \\
\hline & & 3.00 & 1 & $25.0 \%$ & 0 & $0.0 \%$ & 0 & $0.0 \%$ & \\
\hline & & 4.00 & 0 & $0.0 \%$ & 0 & $0.0 \%$ & 1 & $25.0 \%$ & \\
\hline & & 5.00 & 0 & $0.0 \%$ & 1 & $25.0 \%$ & 0 & $0.0 \%$ & \\
\hline & & 6.00 & 0 & $0.0 \%$ & 0 & $0.0 \%$ & 0 & $0.0 \%$ & \\
\hline & \multirow{7}{*}{ Self-etch } & .00 & 1 & $25.0 \%$ & 1 & $25.0 \%$ & 0 & $0.0 \%$ & \multirow{7}{*}{$0.125 \mathrm{NS}$} \\
\hline & & 1.00 & 2 & $50.0 \%$ & 0 & $0.0 \%$ & 0 & $0.0 \%$ & \\
\hline & & 2.00 & 0 & $0.0 \%$ & 2 & $50.0 \%$ & 0 & $0.0 \%$ & \\
\hline & & 3.00 & 1 & $25.0 \%$ & 0 & $0.0 \%$ & 3 & $75.0 \%$ & \\
\hline & & 4.00 & 0 & $0.0 \%$ & 0 & $0.0 \%$ & 0 & $0.0 \%$ & \\
\hline & & 5.00 & 0 & $0.0 \%$ & 1 & $25.0 \%$ & 1 & $25.0 \%$ & \\
\hline & & 6.00 & 0 & $0.0 \%$ & 0 & $0.0 \%$ & 0 & $0.0 \%$ & \\
\hline
\end{tabular}

Means with the same letter within each row are not significantly different at $p=0.05 . \quad$ NS $=$ Non-significant, *=Significant

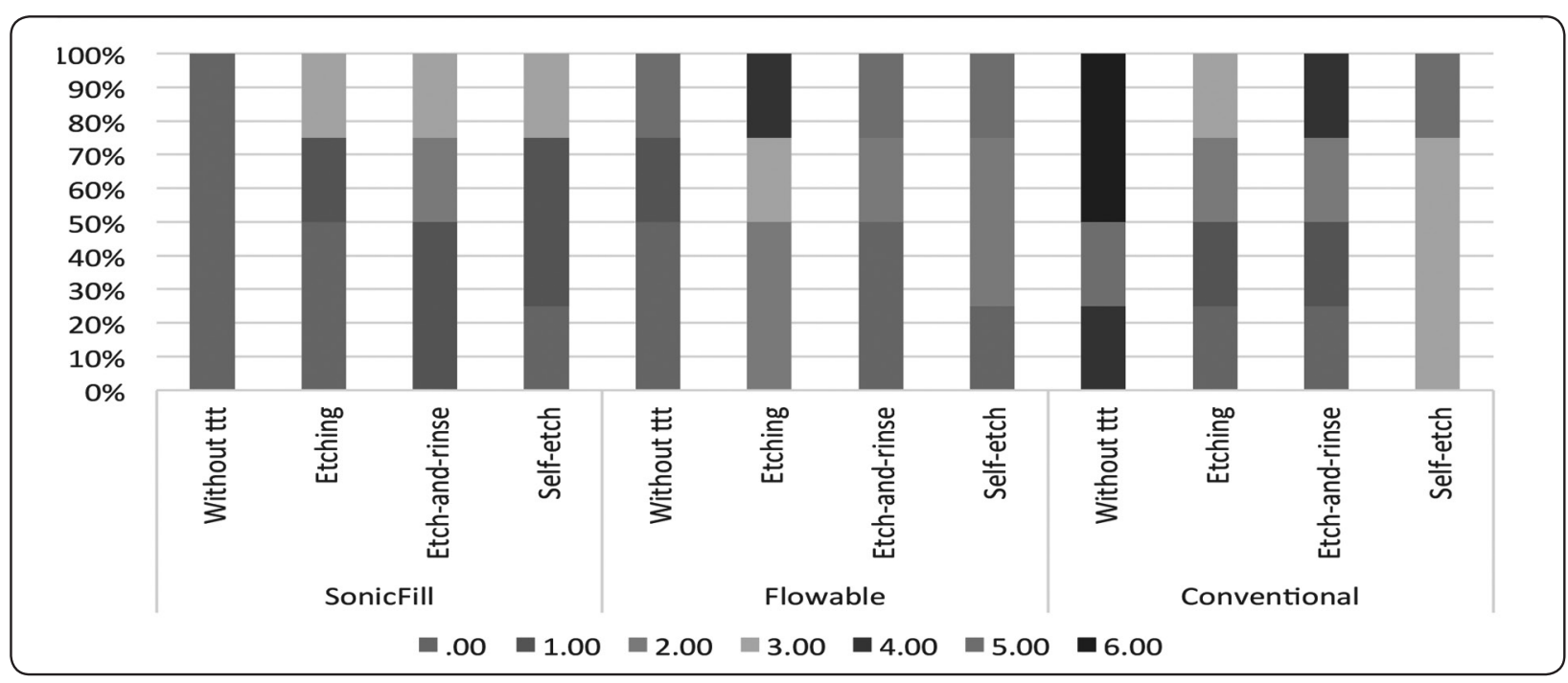

Fig. (1) Histogram showing the percentage of voids count for different repair material within each surface treatment and Substrate condition.

\section{DISCUSSION}

Owing to the growing demands of patients for esthetic restorations, the use of direct, lightcured resin composites in restorative dentistry has markedly expanded. Adhesive dentistry offers the possibility for more conservative treatment, due to the reduction in the size of cavity preparation and bonding of restorative materials to the dental structure. Repair is a viable alternative for replacement as long as the obtained bond strength is close to the cohesive strength of composite. ${ }^{(3)}$ Maximal adaptation void free interface between the repaired material and the old existing composite together with the tooth structure are mandatory for durable and successful final restoration. 
Several factors might influence the adaptation, not only the material itself, but also the surface pretreatment either chemical or mechanical treatments or combinations. There were no statistical significant difference between the different surface treatments and different repair materials in the void analysis except with no surface treatment group which showed a statistical significant difference with $p$ value $<0.005$ with the sonicfill in comparison to flowable and the conventional composite which might be attributed in the effect of the preparation on the adaptation and the void free interface. Opdam et al., in $2003,{ }^{(4)}$ stated that it is impossible to restore a narrow defect with flowable composite without including voids. Concerning the effect of surface roughness and surface treatment on voids formation, it had no effect on voids either within the material or at the interface since it is a function of the material itself and was not affected the substrate condition or the surface treatment. This was in agreement with Schulze et al in 2003, ${ }^{(5)}$ who found that there is a relation between the voids formation and the composite type used but not the pretreatment of the substrate.

\section{CONCLUSION}

\section{Under the limitations of the study, the following Conclusions were observed:}

1. Although flowable composite was characterized by voids in the bulk of the material, interfacial voids were more evident in conventional composite.

2. Surface roughening and chemical pre-treatment had no effect on the internal voids of the repair material; it is rather a function of the repair material itself.

\section{CLINICAL RECOMMENDATIONS}

Void present during the repair procedure is a matter of the material itself and not due to the surface pre-treatment done prior to the repair.

\section{REFERENCES}

1. Demarco FF, Corrêa MB, Cenci MS, Moraes RR, Niek J M Opdam. Longevity of posterior composite restorations: Not only a matter of materials. Dent Mater. 2012; 28(1):87-101.

2. Moncada G, Martin J, Hempel MC, Mjör I a, Gordan V V. A Sealing, Refurbishment and Repair of Class I and Class II Defective Restorations: Three-Year Clinical Trial. J Am Dent Assoc. 2010;140:425-432.

3. Cavalcanti A. De Lima A. Peris A., Mitsui F. MG. Effect of surface treatments and bonding agents on the bond strength of repaired composites. J Esthet Restor Dent. 2007;19(2):90-98.

4. Opdam N, Roeters J, Pesschier T de BD, Bronkhorst E. voids and porosities in class I micropreparations filled with various resin composites. Oper Dent. 2003;28(2):9-14.

5. Schulze K, Zaman A, Söderholm KJ. Effect of filler fraction on strength, viscosity and porosity of experimental compomer materials. J Dent. 2003;31(6):373-382. 